\title{
Association of emergence of new mutations in circulating tumuor DNA during chemotherapy with clinical outcome in metastatic colorectal cancer
}

Ning Jia ${ }^{1}$, Lianpeng Chang ${ }^{2}$, Xin Gao ${ }^{3}$, Xiaohua Shi ${ }^{4}$, Xuelin Dou ${ }^{1}$, Mei Guan ${ }^{1}$, Yajuan Shao ${ }^{1}$, Ningning Li ${ }^{1}$, Yuejuan Cheng ${ }^{1}$, Hongyan Ying ${ }^{1}$, Zhao Sun ${ }^{1}$, Yanping Zhou', Lin Zhao ${ }^{1}$, Jianfeng Zhou ${ }^{1 *}$ and Chunmei Bai ${ }^{* *}$

\begin{abstract}
Background: The understanding of molecular changes in $\mathrm{MCRC}$ during treatment could be used to personalise therapeutic strategies. The aim of our study was to explore the association of circulating tumour DNA (ctDNA) with clinical outcome in metastatic colorectal cancer (mCRC).

Methods: Sequential patients with $\mathrm{mCRC}$ receiving standard first-line chemotherapy were included prospectively. Both plasma ctDNA and serum CEA were assessed in samples obtained before treatment and after 4 cycles of chemotherapy (C4). Computed tomography (CT) scans were carried out at baseline and post-C4 (8-10 weeks) and were assessed using Response Evaluation Criteria In Solid Tumours version 1.1 (RECIST v1.1). Target-capture deep sequencing with a panel covering 1021 genes was performed to detected somatic mutations in ctDNA.

Results: A total of 20 patients were prospectively included and treated with either leucovorin, fluorouracil, and oxaliplatin (FOLFOX) (15/20) or leucovorin, fluorouracil, and irinotecan (FOLFIRI) (5/20). Median follow-up was 6.9 months (range 1.6-26.6). Somatic mutations for baseline ctDNA analysis were identified in $85 \%(17 / 20)$ of the patients. Mutation variations of ctDNA after chemotherapy were tested in 16/20 (80.0\%) of the patients. In multivariate analyses, a high baseline molecular tumour burden index (mTBI) in ctDNA was associated with a higher risk of disease progression, as well as emergence of new mutations in ctDNA during chemotherapy. Patients with newly detected mutations had shorter progression-free survival (PFS) compared to those without (median 3.0 versus 7.3 months; hazard ratio (HR), 5.97; 95\% confidence interval $(\mathrm{Cl}), 0.70-50.69 ; P=0.0003)$. Fold changes in $\mathrm{mTBI}$ from baseline to post-C4 were obtained in $80.0 \%(16 / 20)$ of the patients, which were also related to PFS. Patients with fold reduction in $\mathrm{mTBl}$ above 0.8-fold had longer PFS compared to those below (median 9.3 versus 4.1 months; HR, 4.51; 95\% Cl, 1.29-15.70; $P=0.0008)$.
\end{abstract}

Conclusions: Newly detected mutations in ctDNA during treatment might potentially be associated with clinical outcome in $\mathrm{mCRC}$ and may provide important clinical information.

\footnotetext{
*Correspondence: ZhouJF@pumch.cn; baichunmei1964@163.com

'Department of Medical Oncology, Peking Union Medical College Hospital, Chinese Academy of Medical Sciences and Peking Union Medical College, 1 Shuaifuyuan, Dongcheng District, Beijing 100730, China

Full list of author information is available at the end of the article
} 
Keywords: New mutation, Circulating tumour DNA, Next generation sequencing, Biomarker, Metastatic colorectal cancer

\section{Introduction}

The identification of prognostic biomarkers able to guide treatment decision remains challenging in the management of metastatic colorectal cancer (mCRC) patients. A prognostic marker would help personalise therapeutic strategies. Personalising treatment according to biomarkers might have important clinical utility and lead to improved therapeutic outcome. Therefore, it is crucial to identify prognostic biomarkers in the management of mCRC.

The pattern of genetic alterations in mCRC is a dynamic model affected by several intrinsic and extrinsic factors, including tumour genetic instability and treatment pressure, which is generally assessed in bioptic samples. However, tissue from biopsy material can sometimes be limited, difficult to access or yield poor quality DNA. Additionally, a biopsy can only cover a limited area of the tumour tissue and only represents a glimpse of the tumoural molecular characterisation. In this consideration, circulating tumour DNA (ctDNA) carrying tumour-specific genomic alterations in the peripheral blood can offer significant advantages given that repeatability of blood draw can be performed with relative ease, making it a potential alternative to onerous repeat biopsies [1, 2]. The dynamisms and the heterogeneity of tumours may be detected using this method, which allows for real-time evaluation.

Several studies have demonstrated the prognostic value of identification of mutations in mCRC [3-7], and some studies have demonstrated that emerging new mutations could be detected in ctDNA [8-10]. CtDNA may be used to track clonal evolution and targeted drug responses in mCRC patients [11]. Although the detection of KRAS mutations in ctDNA may be of prognostic value $[12,13]$, the clinical utility of newly detected mutations in ctDNA has been insufficiently explored. Moreover, the use of next generation sequencing (NGS)based techniques might significantly improve the identification of genomic alterations in patients with mCRC.

In this study, by analysis of somatic mutations with a panel covering 1021 genes using the NGS method, we explored the potential association of the detection of new mutations in ctDNA during chemotherapy with poor prognosis in the patients with metastatic colorectal cancer.

\section{Methods}

\section{Study design}

This prospective study recruited patients from Peking Union Medical College Hospital. Eligible patients had Response Evaluation Criteria In Solid Tumours version1.1 (RECIST v1.1) measurable, chemotherapy naïve $\mathrm{mCRC}$, and were to receive standard first-line combination chemotherapy (leucovorin, fluorouracil, and oxaliplatin (FOLFOX) or leucovorin, fluorouracil, and irinotecan (FOLFIRI) with or without targeted therapy. Treatment continued until the establishment of progressive disease (PD) or until the completion of 12 cycles of treatment followed by maintenance with capecitabine alone when the disease was considered complete response (CR), partial response (PR) or stable disease (SD) [14].

Both plasma ctDNA and serum carcinoembryonic antigen (CEA) were assessed in samples obtained before treatment and after 4 cycles of treatment (C4). Computed Tomography (CT) scan of the chest, abdomen and pelvis was performed at baseline and after 4 cycles of chemotherapy (usually 8-10 weeks after starting treatment). These scans were assessed by a single radiologist, and disease response was evaluated as CR, PR, SD or PD according to RECIST v1.1 [14].

We defined right-sided colon cancer as cancer of the cecum and the ascending colon up to the hepatic flexure and the transverse colon. Left-sided colon cancer comprises cancer of the splenic flexure and cancer in regions distal to the splenic flexure, including the rectum.

\section{Ethics statement}

This study was approved by the Medical Ethics Committee of Peking Union Medical College Hospital (ZS-1358) and carried out in accordance with the Helsinki Declaration on experimentation involving human subjects. Twenty patients with pathologically confirmed mCRC in our department at Peking Union Medical Hospital were recruited. All patients signed informed consent.

\section{Sample preparation, storage and DNA extraction}

Peripheral blood was collected in ethylenediaminetetraacetic acid (EDTA) tubes and centrifuged for $10 \mathrm{~min}$ at $1600 \mathrm{~g}$ at $4{ }^{\circ} \mathrm{C}$ within $2 \mathrm{~h}$ of collection. The cell pellets containing peripheral blood lymphocytes (PBLs) were stored at $-20{ }^{\circ} \mathrm{C}$ until further use. The supernatants were further centrifuged at $16,000 \mathrm{~g}$ for $10 \mathrm{~min}$, and plasma was harvested and stored at $-80^{\circ} \mathrm{C}$ until needed. Circulating DNA (cDNA) was extracted from plasma samples with the QIAamp Circulating Nucleic Acid Kit (Qiagen, Hilden, Germany). Genomic DNA (gDNA) were extracted from peripheral blood cells using the QIAamp DNA Blood Mini Kit (Qiagen, Hilden, Germany). Both DNA extractions were performed 
according to the manufacturer's instructions. gDNA was sequenced as the normal control sample [15].

\section{Target capture and NGS}

Sequencing libraries of both cDNA and gDNA were constructed with the KAPA DNA Library Preparation Kit (Kapa Biosystems, Wilmington, MA, USA) according to the manufacturer's protocol. Libraries were hybridised to custom-designed biotinylated oligonucleotide probes (Integrated DNA Technologies, Iowa, IA, USA). Capture probe was designed to cover hot exons or hot regions of 1021 genes frequently mutated in solid tumours (gene list in Table S1). DNA sequencing was performed using the HiSeq 3000 Sequencing System (Illumina, San Diego, CA) with $2 \times 101$-bp paired-end reads.

\section{Sequencing data analysis}

From raw data, terminal adaptor sequences and lowquality reads were removed. BWA (version 0.7.12-r1039) was employed to align the clean reads to the reference human genome (hg19). Picard (version 1.98) was used to mark polymerase chain reaction (PCR) duplicates. Realignment and recalibration was performed using GATK (version 3.4-46-gbc02625). Single nucleotide variants (SNVs) were called using MuTect (version 1.1.4) and NChot, a software developed in-house to review hotspot variants [16]. Small insertions and deletions (indels) were identified by GATK. Somatic copy number alterations were identified with CONTRA (v2.0.8). Significant copy number variation was expressed as the ratio of adjusted depth between ctDNA and control gDNA. The final candidate variants were all manually verified in the Integrative Genomics Viewer (IGV).

\section{Clonal population structure construction and molecular tumour burden index (mTBI)}

PyClone was used to analyse the clonal population structure of ctDNA before treatment and to cluster trunk mutations in each ctDNA collected serially from each patient. mTBI was calculated as the mean allele fraction of trunk mutations in the mutation cluster in each ctDNA [17].

\section{Statistical analysis}

Descriptive statistics and the Mann-Whitney U-test were used to assess the clinical and biochemical variables associated with baseline mTBI. Cox proportional hazards analysis (enter method) was used to estimate the hazard ratios with $95 \%$ confidence intervals for the treatment effect in relation to progression-free survival (PFS) and biomarkers or prognostic clinical information. Multivariate analyses were adjusted for wellcharacterised risk factors: age, gender, Eastern Cooperative Oncology Group (ECOG) performance status (PS), location of primary tumour and metastatic synchronicity. None of these variables was missing. Correlations between the circulating biomarkers (ctDNA and CEA) and tumour response (measured as per RECIST1.1) were assessed using Spearman's rank correlation. The MannWhitney U-test was also used to assess whether there was a significant difference in PFS between groups with and without new mutations in ctDNA. PFS was analysed using the Kaplan-Meier method and compared with the log-rank test. PFS was defined as the time elapsed from the first cycle of treatment until the date of first progression or death (all causes) or censoring at last follow-up. Receiver operating characteristic (ROC) analysis was performed to evaluate the ability of variables to predict PFS and different response. The cut-off values were estimated at various sensitivities and specificities and were determined at the maximum Youden's index. The Mann-Whitney U-test was used to assess whether there was a significant difference in quantitative variables between groups of different responses at first restaging. For qualitative variables, patients were grouped according to the presence or absence of each variable, and Fisher's exact was used to compare variables in relation to tumour response. All statistical analyses were performed with SPSS (v.22.0; STATA, College Station, TX, USA) or GraphPad Prism (v. 7.0; GraphPad Software, La Jolla, CA, USA) software. Statistical significance was defined as a two-sided $P$-value of $<0.05$.

\section{Results}

\section{Patient characteristics}

Twenty patients were prospectively enrolled, all of whom had at least 1 baseline blood draw. None of the patients with RAS wild type in this cohort could afford the expense of additional anti-EGFR monoclonal antibodies that were not covered by insurance back at that time. Figure 1 summarises the flow of patients through the study. At the time of analysis, 17 of the $20(85.0 \%)$ patients evaluable for PFS had experienced disease progression, providing a median PFS of 7.3 months (interquartile range (IQR): 4.1-9.9 months). The characteristics of the patients and association with baseline mTBI are shown in Table 1. The mTBI at baseline was significantly higher for male patients versus female patients $(P=0.0100)$. No significant difference in baseline mTBI was associated with the other patient characteristics tested. The trunk mutations clustered in each ctDNA and its variations after treatment are listed in Table S2.

\section{Detection of ctDNA at baseline and during treatment}

At least 1 mutation was identified in the baseline plasma of $85.0 \%(17 / 20)$ cases. Genomic mutations of ctDNA observed twice or more in the overall cohort at baseline are shown in Fig. S1. There were 40 mutated genes 


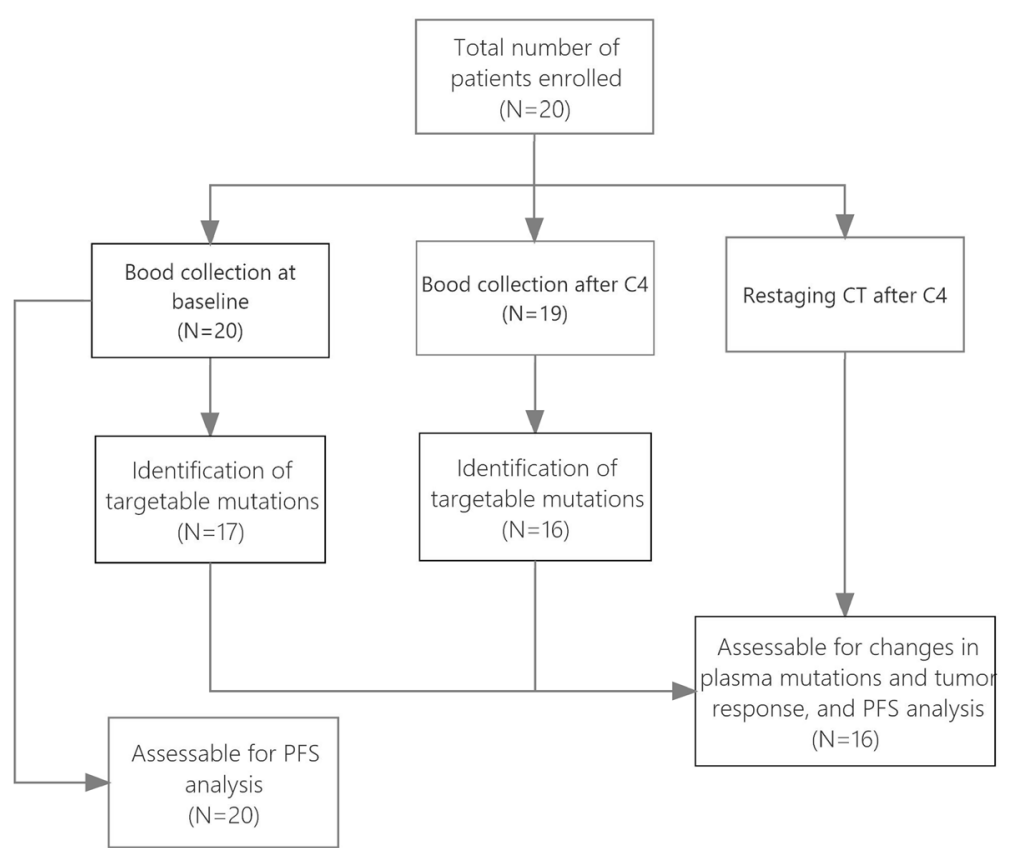

Fig. 1 Diagram showing the flow of patients through the study, including the number of patients included in each of the analysis end points

detected in total. The 3 most frequently mutated genes were TP53 $(94.1 \%, 16 / 17)$, APC $(70.6 \%, 12 / 17)$ and KRAS $(47.1 \%, 8 / 17)$. No regularity of mutation distribution in patients with different age, gender, ECOG PS and location of primary tumour was seen. Blood samples after C4 from 19/20 patients were collected. Mutations in plasma ctDNA after C4 were detectable in 16/19 (84.2\%) patients, 4 of whom emerged 11 new mutated genes as shown in Table 2 . The somatic mutations identified in plasma and the respective abundances of ctDNA mutations and their variations between baseline and after treatment are shown in Table S3.

\section{Concordance of KRAS and BRAF V600E mutation between tumour tissue and matched plasma ctDNA samples}

Nine of the 20 enrolled patients had results of tumoural KRAS status determined by NGS platform-ion torrent PGM and/or amplification refractory mutation system (ARMS). Six of the 20 patients had results of tumoral BRAF V600E status determined by NGS platform-ion torrent PGM and/or real-time polymerase chain reaction (RT-PCR). The KRAS and BRAF V600E status in the matched plasma and tissue from each patient is summarised in Table S4, which was concordant in every case.

\section{Variations in ctDNA during treatment and tumour response}

At the time of analysis, 8 of the 20 patients in this study evaluable for tumour response had partial response with a $40.0 \%$ objective response rate (ORR), including 5 of the 16 patients evaluable for new mutations with a $31.0 \%$ ORR. There was a statistically significant difference in fold change in mTBI after C4 between groups of patients with tumour response and non-response $(P=0.0085$, Fig. S2A). However, no significant difference was seen in fold change in serum CEA $(P=0.0687$, Fig. S2B). Relations between tumour response after $\mathrm{C} 4$ and clinical factors are shown in Table 3. Fold change in mTBI after $\mathrm{C} 4$ was related to tumour response (Mann-Whitney U-test at $P=$ 0.0076). Tumour response was not associated with the other patient characteristics tested. In the 14 patients with reduction of $\mathrm{mTBI}$, the partial response rate and stable disease rate were $35.7 \%(5 / 14)$ and $50.0 \%$ (7/14), respectively. Two patients with progressive disease emerged new mutations in ctDNA during treatment. During the follow-up period, we collected the blood samples of P03 and P04 at the time of disease progression. The mTBI of $\mathrm{P} 03$ reduced from $48.6 \%$ at baseline to $0.0 \%$ at restaging after $\mathrm{C} 4$ with achievement of a partial response, which increased to $43.3 \%$ at the time of disease progression. The mTBI of P04 showed a similar variation tendency to P03: $45.5 \%$ at baseline, $2.1 \%$ at restaging with stable disease and $42.2 \%$ at the time of disease progression. The objective response rate was $41.7 \%(5 / 12)$ and $0.0 \%(0 / 4)$ in the patients without and with emergence of new mutations in ctDNA during treatment, respectively, while there was no statistically significant 
Table 1 Patient characteristics and association with baseline mTBI $(N=20)$

\begin{tabular}{|c|c|c|c|}
\hline Characteristic & $\mathrm{N}(\%)$ & $\begin{array}{l}\text { Baseline mTBI (\%) } \\
\text { Median (IQR) }\end{array}$ & $P$ Value \\
\hline All patients & 20 & & \\
\hline Age (years), median & $60(34-76)$ & & \\
\hline \multicolumn{4}{|l|}{ Age group } \\
\hline$<60$ & $11(55.0)$ & $3.62(0.90-27.54)$ & - \\
\hline $60+$ & $9(45.0)$ & $23.79(4.11-48.52)$ & 0.2601 \\
\hline \multicolumn{4}{|l|}{ Gender } \\
\hline Female & $6(30.0)$ & $3.24(0.00-4.11)$ & - \\
\hline Male & $14(70.0)$ & $38.72(6.80-57.99)$ & 0.0111 \\
\hline \multicolumn{4}{|l|}{ ECOG } \\
\hline 0 & $15(75.0)$ & $5.00(1.24-38.72)$ & - \\
\hline 1 & $5(25.0)$ & $45.52(6.37-73.77)$ & 0.0978 \\
\hline \multicolumn{4}{|l|}{ Primary tumor site } \\
\hline Left-side colon & $16(80.0)$ & $9.01(1.38-44.57)$ & - \\
\hline Right-side colon & $4(20.0)$ & $13.71(3.34-60.85)$ & 0.8198 \\
\hline \multicolumn{4}{|l|}{ Synchronicity of metastasis } \\
\hline Metachrone & $10(50.0)$ & $12.49(1.65-45.9)$ & - \\
\hline Synchrone & $10(50.0)$ & $7.20(2.43-46.3)$ & 1.0000 \\
\hline \multicolumn{4}{|l|}{ Primary tumor(s) } \\
\hline Resected & $15(75.0)$ & $5.00(1.79-38.72)$ & - \\
\hline Unresected & $5(25.0)$ & $41.70(4.70-59.36)$ & 0.3941 \\
\hline \multicolumn{4}{|l|}{ Metastatic site(s) } \\
\hline 1 without peritoneum & $9(45.0)$ & $9.40(2.24-47.02)$ & - \\
\hline $2+$ without peritoneum & $11(55.0)$ & $8.62(1.79-38.72)$ & 0.8817 \\
\hline Peritoneum & 0 & & - \\
\hline \multicolumn{4}{|l|}{ Baseline serum CEA } \\
\hline Normal & $5(25.0)$ & $1.79(0.62-13.52)$ & - \\
\hline Elevated & $15(75.0)$ & $16.36(4.11-48.52)$ & 0.0522 \\
\hline \multicolumn{4}{|l|}{ Chemotherapy regimen } \\
\hline FOLFOX & $15(75.0)$ & $8.62(3.24-45.52)$ & - \\
\hline FOLFIRI & $5(25.0)$ & $16.36(1.52-51.94)$ & 0.9327 \\
\hline \multicolumn{4}{|l|}{ Bevacizumab received } \\
\hline Yes & $1(5.0)$ & 23.79 & - \\
\hline No & $19(95.0)$ & $8.62(1.79-45.52)$ & - \\
\hline
\end{tabular}

$P$ value indicates a significance level of $<0.05$

$I Q R$ interquartile range, ECOG Eastern Cooperative Oncology Group performance status, CEA carcinoembryonic antigen

difference in ORR between patients with and without emergence of new mutations in ctDNA during treatment (Fisher's exact at $P=0.0885$ ).

\section{Baseline ctDNA and PFS}

Predictive value of clinical factors, including age, gender, ECOG PS, location of primary tumour, synchronicity of metastasis, serum CEA at baseline, mTBI at baseline and identification of new mutations during chemotherapy was evaluated. In univariate analyses, we observed that the high mTBI level at baseline recorded as a continuous variable was significantly associated with a shorter PFS $(P=0.0494)$, as well as for patients with emergence of new mutations in ctDNA during treatment versus those without mutations $(P=0.008)$. In multivariate analyses, the high mTBI level at baseline remained significantly associated with a shorter PFS $(P=0.0084)$, as well as for male patients versus female patients $(P=0.0204)$, patients with ECOG PS 1 versus ECOG PS $0(P=0.0455)$, patients with synchronous versus metachronous metastatic disease $(P=0.0423)$ and patients with emergence of new mutations in ctDNA during treatment versus those without $(P=0.0033)$, which are shown in Table 4 . Negative correlation between the baseline mTBI and PFS was seen in this cohort of patients (Spearman correlation, $P=0.0083, r=-0.5725$; Fig. 2a). The optimal baseline $\mathrm{mTBI}$ for predicting PFS, as determined by the ROC curves (ROC area $=0.83, P=0.0126$ ), was $6.8 \%$. Patients with baseline mTBI below $6.8 \%$ had longer PFS compared to those above (median 9.9 versus 4.35 months; hazard ratio (HR), 2.97; 95\% confidence interval (CI), 1.08-8.18; $P=0.0115$; Fig. 3a). There was no significant association of the baseline serum CEA level with PFS $(P=0.7363)$. No correlation between the baseline serum CEA level and PFS was observed (Spearman correlation, $P=0.2928, r=-0.2475$; Fig. $2 b)$.

Identification of new mutations during treatment and PFS The patient group with identification of new mutations during treatment had a significantly shorter PFS than the patient group without identification of new mutations (median 3.0 versus 7.3 months; HR, 5.97; 95\% CI, 0.70-50.69; $P=0.0003$; Fig. 3b).

\section{CtDNA variation and PFS}

In univariate analyses, the association of fold reduction of mTBI with PFS was observed (HR, 2.92, 95\% CI, 1.17-7.28; $P=0.0214)$. In multivariate analyses, patients who had a more fold reduction of mTBI had a significantly longer PFS than patients with less or no fold reduction of mTBI after adjustment for age, gender, ECOG PS, location of primary tumour and metastatic synchronicity (HR, 27.27, 95\% CI, 1.49-498.10; $P=$ 0.0257 , Table S5). The optimal fold reduction in $\mathrm{mTBI}$ for predicting PFS, as determined by the ROC curves (ROC area $=0.8438, P=0.0209$ ), was 0.8 -fold. Patients with fold reduction in mTBI above 0.8 -fold had longer PFS compared to those below (median 9.3 versus 4.1 months; HR, 4.51; 95\% CI: 1.29-15.70; $P=0.0008$, Fig. 3c). In contrast, serum CEA level variation did not correlate with PFS. 
Table 2 New mutations detected in plasma ctDNA during treatment

\begin{tabular}{|c|c|c|c|c|c|c|}
\hline Patient ID & Time point & New mutation & cHGVS & pHGVS & Function & VAFs (\%) \\
\hline P06 & Post-C2 & KRAS & c. $38 \mathrm{G}>\mathrm{A}$ & p.G13D & missense & 0.36 \\
\hline \multirow[t]{2}{*}{ P07 } & Post-C4 & CDK13 & c.604_605insT & p.R202Lfs*68 & frameshift & 1.10 \\
\hline & Post-C4 & MTOR & $c .617 G>A$ & p.R206H & missense & 0.73 \\
\hline \multirow[t]{4}{*}{ P08 } & Post-C4 & BRCA2 & c. $6232 \mathrm{G}>\mathrm{A}$ & p.G2078R & missense & 0.90 \\
\hline & Post-C4 & MYC & c. $1211 A>G$ & p.K404R & missense & 0.32 \\
\hline & Post-C4 & FGFR2 & c. $269 \mathrm{~A}>\mathrm{T}$ & p.E90V & missense & 0.56 \\
\hline & Post-C4 & EGFR & c. $2327 \mathrm{G}>\mathrm{A}$ & p.R776H & missense & 0.25 \\
\hline \multirow[t]{4}{*}{ P10 } & Post-C4 & PIK3R2 & $c .451 C>T$ & p.P151S & missense & 1.23 \\
\hline & Post-C4 & DNMT3A & c. $2077 C>$ T & p.R693C & missense & 0.57 \\
\hline & Post-C4 & NTRK1 & C.1859G > A & p.C620Y & missense & 0.60 \\
\hline & Post-C4 & MLL & c. $10480 A>G$ & p.N3494D & missense & 0.60 \\
\hline
\end{tabular}

cHGVS Coding DNA reference sequences (Human Genome Variation Society), pHGVS Protein level amino acid sequences (Human Genome Variation Society), PostC2 After 2 cycles of chemotherapy, Post-C4 After 4 cycles of chemotherapy

\section{Discussion}

Our study shows that ctDNA can be identified in a high proportion of patients with treatment-naive mCRC. The evaluation of a panel of 1021 genes allowed us to identify mutations in the plasma at baseline of 17 cases $(85 \%$ of all 20 patients). Published studies of ctDNA in the patients with mCRC have used varying analytical methods or targeted gene panels which can make data interpretation and comparison across studies difficult. In previous studies, the rate of ctDNA detected in the plasma varied from 73.7 to $90.6 \%$ of patients with mCRC [1, 18-21]. Due to constant improvements in sensitivity of technologies, including those to detect somatic changes not identified by the current approach, the rate of detection might increase continuously, which could make ctDNA more applicable in clinical practice.

Our study also demonstrates the high concordance of ctDNA and tumour tissues for the molecular characterisation (KRAS and BRAF status), which was shown in many previous studies [1, 20, 22-24]. As serial biopsies in $\mathrm{mCRC}$ are not feasible, ctDNA which appears to be a potential surrogate of both tumour molecular profile

Table 3 Relations between tumor response and clinical factors

\begin{tabular}{ll}
\hline Variable & $\boldsymbol{P}$ value \\
\hline Age & 0.3338 \\
Gender & 0.2615 \\
ECOG & 1.0000 \\
Location & 1.0000 \\
Synchronicity & 0.5962 \\
Fold change in CEA & 0.0687 \\
Fold change in mTBI & 0.0076 \\
New mutations & 0.0885 \\
\hline
\end{tabular}

and its heterogeneity, might overcome this limitation in routine clinical practice.

Truncal mutations that represent the majority of tumour lesions in a patient are potentially the best candidates for monitoring tumour burden using ctDNA [25]. In our study, both mTBI at baseline and fold change in mTBI, which was calculated on truncal mutations in ctDNA, are associated with PFS in patients with mCRC. The patients with higher mTBI at baseline in this cohort were nearly 3 times more likely to experience disease progression than the patients with lower mTBI. The patients with reduction in mTBI after treatment had a $78 \%$ lower risk of disease progression than the patients without. Our study shows the potential of using plasma DNA for molecular stratification, which still need to be confirmed in a larger cohort of patients.

Achievement of response to treatment is one of the aims of anti-tumour therapy. Our data showed that fold change in ctDNA after $\mathrm{C} 4$ was related to tumour response. Inexplicably, 2 patients with reduction in ctDNA after 4 cycles of treatment experienced disease progression instead of response to therapy. Subsequently, the ctDNA of the other 2 patients with disease control at first restaging reduced after $\mathrm{C} 4$, and rose up at the time of disease progression during the follow-up period. According to the high concordance of variation tendency of ctDNA and response evaluation in these 2 patients, we proposed that the reduction in ctDNA in the 2 patients with disease progression at the first restaging could have reached its nadir before the first restaging and then increased but remained at a lower level than the baseline value at the first restaging, resulting in the illogical mismatch between the reduction in ctDNA and tumour response. Therefore, serial ctDNA monitoring at more time-points might have better accuracy to predict radiological tumour response which need to be validated in further studies. 
Table 4 Univariate and multivariate analyses of factors for progression-free survival using cox regression model $(N=16)$

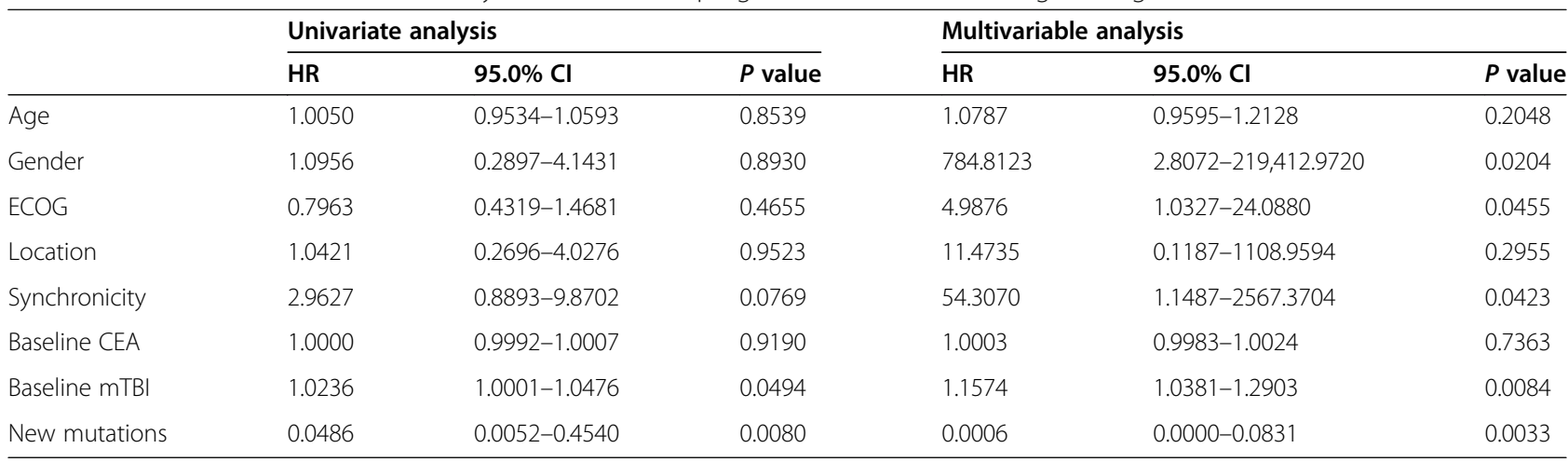

ECOG Eastern Cooperative Oncology Group performance status, CEA carcinoembryonic antigen, $m T B I$ molecular tumour burden index

Our study on mCRC suggests that the detection of new mutations in circulating tumour DNA during treatment may be associated with shorter PFS. The patients with identification of new mutations in this cohort were nearly 6 times more likely to experience disease progression than the patients without, none of whom had tumour response at first restaging. Detection of freecirculating tumour-associated DNA may help the evaluation of colorectal cancer prognosis [12]. Mutational processes evolve across a cancer's lifespan. Although the dynamics of somatic evolution of CRC remain unclear, those somatic mutations conferring a selective advantage on the cell drive successive waves of clonal expansion with therapeutic resistance, which may correlate with cancer progression. Some mutations in tumour tissue have been validated as prognostic biomarkers in CRC. A negative prognostic effect of gene mutation has been confirmed in a large pooled analysis from the PETACC8 and N0147 trials, where KRAS exon 2 and BRAF mutations were identified as independent predictors of shorter time to recurrence and overall survival (OS) among patients with stage III microsatellite stable (MSS) colorectal cancer [26]. In a meta-analysis investigating the impact of KRAS mutation on outcomes in mCRC patients undergoing liver resection, KRAS mutations were negatively associated with $\mathrm{OS}(\mathrm{HR}=2.24)$ and relapse-free survival $(H R=1.89)$ [3]. In multiple previous studies, BRAF mutation has gained a prominent role as a negative prognostic factor since $\mathrm{mCRC}$ patients with BRAF mutation showed shorter median OS $[4,6,7]$. Because of the high concordance of ctDNA and tumour tissues for somatic mutations in the patients with mCRC, mutational evolution in ctDNA may be associated with the prognosis of mCRC. Furthermore, the REVERCE study showed emerging new gene alterations in ctDNA associated with worse survival outcomes [27], which was also shown in this study.

The need for improved molecular stratification of $\mathrm{mCRC}$ is important as the identification of the most effective treatment for an individual patient is still mainly based on clinical considerations, such as symptoms, performance status, extent of disease, patients' preferences and treatment history, while the identification of predictive and/or prognostic biomarkers able to guide treatment decision still stands as a challenging issue in the management of mCRC patients.
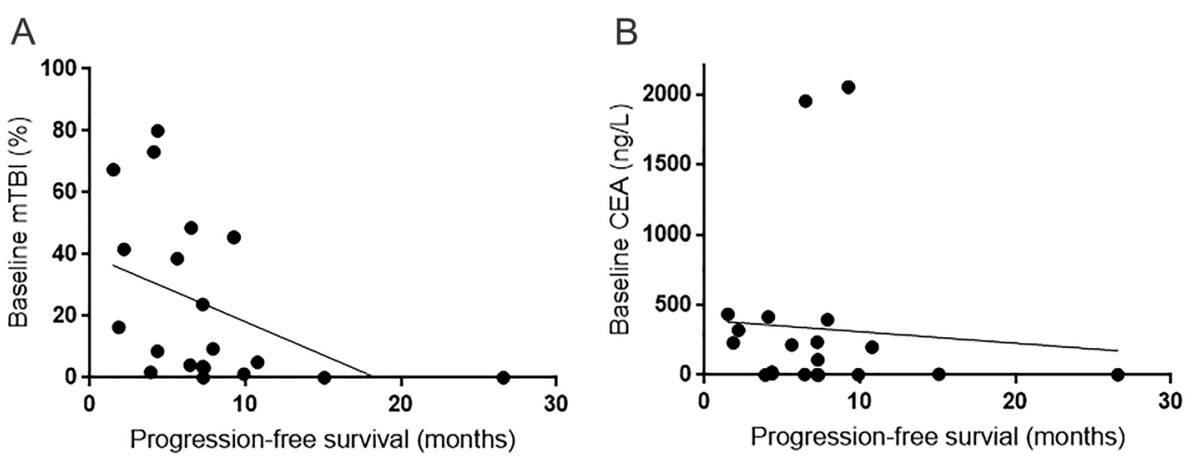

Fig. 2 Correlations between baseline mTBI and CEA level and PFS. a Negative correlation between the baseline mTBI level and PFS was seen: Spearman correlation at $P=0.0083, r=-0.5725$. b No correlation between the baseline serum CEA level and PFS was observed: Spearman correlation at $P=0.2928, r=-0.2475$ 


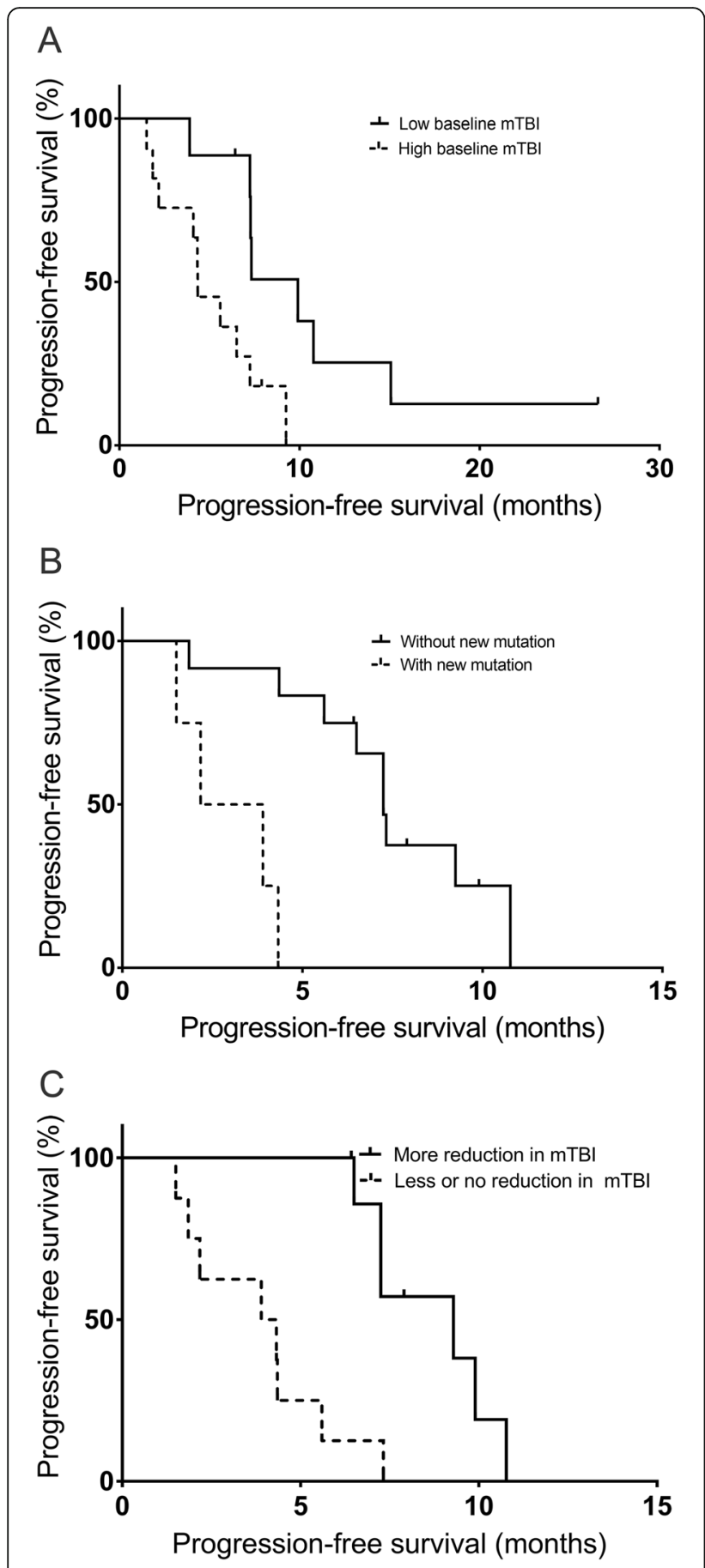

Fig. 3 Kaplan-Meier survival plots of progression-free survival (PFS) in different patient groups in this cohort. a Patients with baseline mTBI level below $6.81 \%$ had longer PFS compared to those above $(P=0.0115)$. b Patients with identification of new mutations during treatment had a significant shorter PFS than the patients without identification of new mutations ( $P=0.0003$ ). c Patients with fold reduction in $\mathrm{mTBI}$ above 0.8 -fold had longer PFS compared to those below $(P=0.0115)$
It is important to understand that molecular profiles are dynamic and might change under treatment pressure. The development of liquid biopsies makes us able to molecularly monitor our patients in real time and to identify molecular mechanism of drug resistance informing us of possible novel treatment options. Our data suggest that patients with high risk of disease progression might potentially be distinguished by the analysis of ctDNA. However, this is a single-center exploratory study with a small sample size. Our data are insufficient to draw robust conclusions. Further work is needed firstly to better understand the mechanisms of association between ctDNA and clinical outcome and secondly to confirm these preliminary results.

\section{Abbreviations}

CtDNA: Circulating tumour DNA; mCRC: Metastatic colorectal cancer; $C T$ : Computed tomography; RECIST: Response evaluation criteria in solid tumours; PD: Progressive disease; CR: Complete response; PR: Partial response; SD: Stable disease; CEA: Carcinoembryonic antigen; C4: Four cycles of treatment; EDTA: Ethylenediaminetetraacetic acid; PBLs: Peripheral blood lymphocytes; CDNA: Circulating DNA; gDNA: Genomic DNA; PCR: Polymerase chain reaction; IGV: Integrative genomics viewer; mTBI: Molecular tumour burden index; PFS: Progression-free survival; ECOG: Eastern cooperative oncology group; PS: Performance status; ROC: Receiver operating characteristic; IQR: Interquartile range; ARMS: Amplification refractory mutation system; RT-PCR: Real-time polymerase chain reaction; ORR: Objective response rate; OS: Overall survival; MSS: Microsatellite stable

\section{Supplementary Information}

The online version contains supplementary material available at https://doi. org/10.1186/s12885-021-08309-2.

Additional file 1 : Table S1. Gene list of 1021 gene panel.

Additional file $\mathbf{2}$ : Table S2. Abundance of trunk clone and its variations between baseline and after 4 cycles of chemotherapy.

Additional file 3 : Supplementary Fig. S1. Landscape of genomic mutations in ctDNA observed twice or more in the overall cohort at baseline. Each column represents an individual patient. The top panel shows the molecular tumor burden. The middle panel presents the frequently mutated genes observed twice or more in our cohort study, and the frequency and function are shown on the left and right, respectively. The bottom panel presents patients' information including age, gender, ECOG, tumor location and stage.

Additional file 4 : Table S3. Abundances of ctDNA mutations and their variations between baseline and during treatment.

Additional file $\mathbf{5}$ : Supplementary Fig. S2. The differences in fold changes in CEA and ctDNA between tumor response and non-response patients. A, Significant difference in fold change in $\mathrm{mTBl}$ after $\mathrm{C} 4$ between groups of patients with tumor response and non-response was observed: Mann-Whitney $U$-test at $P=0.0085$. B, No significant difference was seen in fold change in serum CEA between groups of patients with tumor response and non-response: Mann-Whitney U-test at $P=0.0687$.

Additional file $\mathbf{6}$ : Table S4. Identification of gene status in tumour tissue and baseline ctDNA.

Additional file 7 : Table S5: Multivariate Cox model assessing the impact on progression free survival of ctDNA variations observed between baseline and post-C4 after adjustment on the following variables: age, gender, Eastern Cooperative Oncology Group Performance Status, location of primary tumor and synchronicity of metastatic disease. 


\section{Authors' contributions}

$\mathrm{NJ}$ led analysis and writing of the manuscript. JZ and CB both equally provided major contribution in revising the manuscript and supervised the research. $L C, X D, Y Z$, and $Z S$ developed lab protocols, processed samples, and analysed data. XG, XS, MG, YS, NL, YC, HY and LZ collected, analysed and interpreted the clinical data and outcome. All authors read and approved the final manuscript.

\section{Funding}

This study was supported by grants from CAMS Innovation Fund for Medical Sciences (No. 2016-12M-1-001).

\section{Availability of data and materials}

The authenticity of this article has been validated by uploading the key raw data onto the Research Data Deposit public platform (www.researchdata.org cn), with the approval RDD number as RDDA2021001965.

\section{Declarations}

\section{Ethics approval and consent to participate}

This study was approved by the Medical Ethics Committee of Peking Union Medical College Hospital (ZS-1358) and carried out in accordance with the Helsinki Declaration on experimentation involving human subjects. All patients signed informed consent.

\section{Consent for publication}

Not applicable.

\section{Competing interests}

LC is an employee of Geneplus-Beijing (Beijing, China). The other authors have declared no competing interest.

\begin{abstract}
Author details
'Department of Medical Oncology, Peking Union Medical College Hospital, Chinese Academy of Medical Sciences and Peking Union Medical College, Shuaifuyuan, Dongcheng District, Beijing 100730, China. ${ }^{2}$ Geneplus-Beijing, Beijing, China. ${ }^{3}$ Department of Radiology, Peking Union Medical College Hospital, Chinese Academy of Medical Sciences and Peking Union Medical College, Beijing, China. ${ }^{4}$ Department of Pathology, Peking Union Medical College Hospital, Chinese Academy of Medical Sciences and Peking Union Medical College, Beijing, China
\end{abstract}

Received: 9 January 2021 Accepted: 6 May 2021

Published online: 22 July 2021

\section{References}

1. Bettegowda C, Sausen M, Leary RJ, Kinde I, Wang Y, Agrawal N, et al. Detection of circulating tumor DNA in early- and late-stage human malignancies. Sci Transl Med. 2014;6:224ra24.

2. Newman AM, Bratman SV, To J, Wynne JF, Eclov NC, Modlin LA, et al. An ultrasensitive method for quantitating circulating tumor DNA with broad patient coverage. Nat Med. 2014;20(5):548-54. https://doi.org/10.1038/nm.3 519.

3. Brudvik KW, Kopetz SE, Li L, Conrad C, Aloia TA, Vauthey JN. Meta-analysis of KRAS mutations and survival after resection of colorectal liver metastases. $\mathrm{Br}$ J Surg. 2015;102(10):1175-83. https://doi.org/10.1002/bjs.9870.

4. Richman SD, Seymour MT, Chambers P, Elliott F, Daly CL, Meade AM, et al. KRAS and BRAF mutations in advanced colorectal cancer are associated with poor prognosis but do not preclude benefit from oxaliplatin or irinotecan: results from the MRC FOCUS trial. J Clin Oncol. 2009;27(35):59317. https://doi.org/10.1200/JCO.2009.22.4295.

5. Saridaki Z, Papadatos-Pastos D, Tzardi M, Mavroudis D, Bairaktari E, Arvanity $\mathrm{H}$, et al. BRAF mutations, microsatellite instability status and cyclin D1 expression predict metastatic colorectal patients' outcome. Br J Cancer. 2010;102(12):1762-8. https://doi.org/10.1038/sj.bjc.6605694.

6. Souglakos J, Philips J, Wang R, Marwah S, Silver M, Tzardi M, et al. Prognostic and predictive value of common mutations for treatment response and survival in patients with metastatic colorectal cancer. $\mathrm{Br} \mathrm{J}$ Cancer. 2009;101(3):465-72. https://doi.org/10.1038/sj.bjc.6605164.

7. Yokota T, Ura T, Shibata N, Takahari D, Shitara K, Nomura M, et al. BRAF mutation is a powerful prognostic factor in advanced and recurrent colorectal cancer. Br J Cancer. 2011;104(5):856-62. https://doi.org/10.1038/ bjc.2011.19.

8. Diaz LA Jr, Williams RT, Wu J, Kinde I, Hecht JR, Berlin J, et al. The molecular evolution of acquired resistance to targeted EGFR blockade in colorectal cancers. Nature. 2012;486(7404):537-40. https://doi.org/10.1038/nature11219.

9. Misale S, Yaeger R, Hobor S, Scala E, Janakiraman M, Liska D, et al. Emergence of KRAS mutations and acquired resistance to anti-EGFR therapy in colorectal cancer. Nature. 2012;486(7404):532-6. https://doi.org/10.1038/ nature11156.

10. Laurent-Puig P, Pekin D, Normand C, Kotsopoulos SK, Nizard P, Perez-Toralla $K$, et al. Clinical relevance of KRAS-mutated subclones detected with picodroplet digital PCR in advanced colorectal cancer treated with antiEGFR therapy. Clin Cancer Res. 2015;21(5):1087-97. https://doi.org/10.1158/1 078-0432.CCR-14-0983.

11. Siravegna G, Mussolin B, Buscarino M, Corti G, Cassingena A, Crisafulli G, et al. Clonal evolution and resistance to EGFR blockade in the blood of colorectal cancer patients. Nat Med. 2015:21(7):795-801. https://doi.org/10.1 038/nm.3870

12. Lecomte T, Berger A, Zinzindohoue F, Micard S, Landi B, Blons $\mathrm{H}$, et al. Detection of free-circulating tumor-associated DNA in plasma of colorectal cancer patients and its association with prognosis. Int J Cancer. 2002;100(5): 542-8. https://doi.org/10.1002/ijc.10526.

13. Wong AL, Lim JS, Sinha A, Gopinathan A, Lim R, Tan CS, et al. Tumour pharmacodynamics and circulating cell free DNA in patients with refractory colorectal carcinoma treated with regorafenib. J Transl Med. 2015;13(1):57. https://doi.org/10.1186/s12967-015-0405-4.

14. Jia N, Sun Z, Gao X, Cheng Y, Zhou Y, Shen C, et al. Serial monitoring of circulating tumor DNA in patients with metastatic colorectal cancer to predict the therapeutic response. Front Genet. 2019;10:470. https://doi.org/1 0.3389/fgene.2019.00470.

15. Zhou J, Chang L, Guan Y, Yang L, Xia X, Cui L, et al. Application of circulating tumor DNA as a non-invasive tool for monitoring the progression of colorectal cancer. PLoS One. 2016;11(7):e0159708. https://doi. org/10.1371/journal.pone.0159708.

16. Yang $X$, Chu $Y$, Zhang $R$, Han $Y$, Zhang $L$, Fu Y, et al. Technical validation of a next-generation sequencing assay for detecting clinically relevant levels of breast cancer-related single-nucleotide variants and copy number variants using simulated cell-free DNA. J Mol Diagn. 2017;19(4):525-36. https://doi. org/10.1016/j.jmoldx.2017.04.007

17. Ma F, Guan Y, Yi Z, Chang L, Xia X, Li Q, et al. Assessing tumor heterogeneity using circulating tumor DNA to predict and monitor therapeutic response in metastatic breast cancer. J Clin Oncol. 2017;35: 11543.

18. Taly V, Pekin D, Benhaim L, Kotsopoulos SK, Le Corre D, Li X, et al. Multiplex picodroplet digital PCR to detect KRAS mutations in circulating DNA from the plasma of colorectal cancer patients. Clin Chem. 2013;59(12):1722-31. https://doi.org/10.1373/clinchem.2013.206359.

19. Spindler KL, Pallisgaard N, Andersen RF, Jakobsen A. Changes in mutational status during third-line treatment for metastatic colorectal cancer--results of consecutive measurement of cell free DNA, KRAS and BRAF in the plasma. Int J Cancer. 2014;135(9):2215-22. https://doi.org/10.1002/ijc.28863.

20. Tie J, Kinde I, Wang Y, Wong HL, Roebert J, Christie M, et al. Circulating tumor DNA as an early marker of therapeutic response in patients with metastatic colorectal cancer. Ann Oncol. 2015;26(8):1715-22. https://doi. org/10.1093/annonc/mdv177.

21. Garlan F, Laurent-Puig P, Sefrioui D, Siauve N, Didelot A, Sarafan-Vasseur N, et al. Early evaluation of circulating tumor DNA as marker of therapeutic efficacy in metastatic colorectal cancer patients (PLACOL study). Clin Cancer Res. 2017;23(18):5416-25. https://doi.org/10.1158/1078-0432.CCR-16-3155.

22. Morgan SR, Whiteley J, Donald E, Smith J, Eisenberg MT, Kallam E, et al. Comparison of KRAS mutation assessment in tumor DNA and circulating free DNA in plasma and serum samples. Clin Med Insights Pathol. 2012:5: 15-22. https://doi.org/10.4137/CPath.S8798.

23. Thierry AR, Mouliere F, El Messaoudi S, Mollevi C, Lopez-Crapez E, Rolet F, et al. Clinical validation of the detection of KRAS and BRAF mutations from circulating tumor DNA. Nat Med. 2014:20(4):430-5. https://doi.org/10.1038/nm.3511.

24. Schmiegel W, Scott RJ, Dooley S, Lewis W, Meldrum CJ, Pockney P, et al. Blood-based detection of RAS mutations to guide anti-EGFR therapy in colorectal cancer patients: concordance of results from circulating tumor DNA and tissue-based RAS testing. Mol Oncol. 2017;11(2):208-19. https:// doi.org/10.1002/1878-0261.12023. 
25. Murtaza M, Dawson SJ, Pogrebniak K, Rueda OM, Provenzano E, Grant J, et al. Multifocal clonal evolution characterized using circulating tumour DNA in a case of metastatic breast cancer. Nat Commun. 2015;6(1):8760. https://doi.org/10.1038/ncomms9760.

26. Taieb J, Malicot KL, Penault-Llorca FM, Bouche O, Shi Q, Thibodeau SN, et al. Prognostic value of BRAF V600E and KRAS exon 2 mutations in microsatellite stable (MSS), stage III colon cancers (CC) from patients (pts) treated with adjuvant FOLFOX+/- cetuximab: a pooled analysis of 3934 pts from the PETACC8 and N0147 trials. J Clin Oncol. 2015;33:3507.

27. Shitara K, Yamanaka T, Denda T, Tsuji Y, Shinozaki K, Komatsu Y, et al. REVE RCE: a randomized phase II study of regorafenib followed by cetuximab versus the reverse sequence for previously treated metastatic colorectal cancer patients. Ann Oncol. 2019;30(2):259-65. https://doi.org/10.1093/a nnonc/mdy526.

\section{Publisher's Note}

Springer Nature remains neutral with regard to jurisdictional claims in published maps and institutional affiliations.

Ready to submit your research? Choose BMC and benefit from:

- fast, convenient online submission

- thorough peer review by experienced researchers in your field

- rapid publication on acceptance

- support for research data, including large and complex data types

- gold Open Access which fosters wider collaboration and increased citations

- maximum visibility for your research: over $100 \mathrm{M}$ website views per year

At $\mathrm{BMC}$, research is always in progress.

Learn more biomedcentral.com/submissions 